\title{
CORRIGENDUM
}

\section{Downregulation of ZEB1 and overexpression of Smad7 contribute to resistance to TGF- $\beta 1$-mediated growth suppression in adult T-cell leukemia/lymphoma}

\author{
S Nakahata, S Yamazaki, H Nakauchi and K Morishita
}

Oncogene (2011) 30, 2900; doi:10.1038/onc.2011.158

Correction to: Oncogene (2010) 29, 4157-4169; doi:10.1038/onc.2010.172; published online 31 May 2011

Since the publication of the above paper, the authors have identified three errors in the Supplemental materials and methods section of the paper. First, the correct primer used in the ChIP experiments should be murine $\beta$-actin reverse primer $5^{\prime}$-GAATAGCCTCCGCCCTT GT $-3^{\prime}$. The primer sequence was inadvertently a copy of the murine p21 reverse primer used in the same ChIP experiments. Second, the authors identified an incorrect shRNA sequence that targets Smad7. The correct sequence should be $5^{\prime}$-GTTTCTCCATCAAGGCTTT-3'. This error occurred while quoting part of a sentence from a paper that had been previously published in International Immunology, Vol. 20, No. 11, pp. 1417-1426. Third, the shRNA sequence against murine ZEB1 was missing. The target sequence was as follows: $5^{\prime}$-CCTGTGGATT ATGAGTTCA-3'. The authors would like to apologize for these mistakes. 